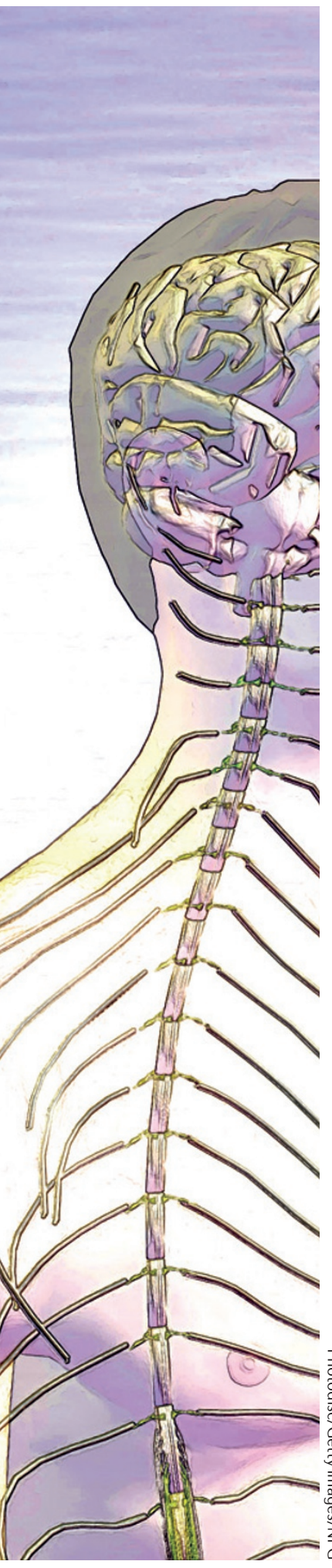

DISEASE GENETICS

\title{
tRNA splicing defect underlies brain disorder
}

In a great example of how international collaborations can advance biomedical research, two back-to-back publications in Cell define a novel clinical syndrome that affects both the peripheral and the central nervous system, and that arises from mutations in CLP1, which encodes a mammalian RNA kinase that forms part of the tRNA splicing endonuclease (TSEN) complex. Both studies implicate defective tRNA splicing as the underlying molecular cause of the syndrome, thus adding to a growing body of literature that links neurological diseases with tRNA modifications.

Large-scale whole-exome sequencing of children with signs of neurodevelopmental disorders — including microcephaly, epilepsy, sensory and motor defects, and intellectual disability — identified Turkish families in which affected family members were homozygous for the same single-nucleotide substitution in the CLP1 gene. In both studies, the exact features that constitute the clinically distinguishable phenotype only became evident after the mutation had been discovered, which enabled the researchers to define the disease. Additionally, in the cohort sequenced by James R. Lupski (Baylor College of Medicine) and co-workers, distinction of shared clinical features in patients with the CLP1 mutation helped to identify additional affected families. "The interesting thing about this approach is that it reverses the usual paradigm of 'gene hunting,' says Joseph G. Gleeson (University of California, San Diego), who led the second study. "Starting with a group of patients, their DNA defines the clinical features, rather than the doctors."
The CLP1 mutation leads to an Arg140His amino acid substitution that both teams predicted would change the protein structure. To elucidate the cellular consequences of this mutation, Gleeson and colleagues used patient-derived skin fibroblasts and induced their transdifferentiation into neurons by knockdown of polypyrimidine tract-binding protein 1 (PTBP1). In both cell types, in vitro kinase activity and nuclear localization of the CLP1 protein were severely disrupted. Meanwhile, Lupski had joined forces with teams led by Josef M. Penninger and Javier Martinez (Austrian Academy of Sciences, Vienna), and showed using in vitro assays that the kinase activity of mutant CLP1, albeit reduced, was still sufficient to phosphorylate RNA. However, they identified an impaired interaction of the mutant with TSEN proteins that correlated with decreased pre-tRNA splicing. The same effect on tRNA biogenesis was anticipated by Gleeson and colleagues. "The CLP1 mutation, we predicted, should cause a backlog of pre-tRNAs and depletion of mature tRNAs," remembers Gleeson. "Indeed, we found that patient-derived induced neurons, but not fibroblasts, showed this defect, which points to a potential use of these cells for other nervous system-specific diseases."

At the same time, the findings in humans prompted Penninger and his team to undertake further analyses of previously generated mice with a single amino acid change that abolishes CLP1 kinase activity. These animals had been shown to develop some, but not all, of the neurological defects exhibited by patients with the CLP1 mutation, for example, progressive loss of motor neurons. "To study brain size we used the strongest MRI on the planet, a 15.2 tesla MRI, and the resulting images are the first ones ever to be published with this field strength," says Penninger. These detailed analyses revealed another similarity between the mouse model and the patients: microcephaly. This finding had been missed upon initial characterization of the mice, which means that the human phenotype drove this discovery.

"Both the central and the peripheral nervous system being affected by disease suggested that early progenitor cells, perhaps stem cells, were involved," says Lupski. Indeed, the reduced brain volume in mice resulted from a decrease in the number of cortical neurons owing to increased cell death of neural progenitor cells. Overall, the findings suggest that the subtle perturbation of basic cellular processes, such as tRNA biogenesis, could lead to a susceptibility of neuroprogenitor cells to apoptosis. "Knowing fundamental pathways that regulate degeneration of neurons should allow us to define new pathways that, when modulated, might help us to protect motor neurons from dying, such as in amyotrophic lateral sclerosis," concludes Penninger.

Linda Koch

ORIGINAL RESEARCH PAPERS Schaffer, A. E. et al. CLP1 founder mutation links tRNA splicing and maturation to cerebellar development and neurodegeneration. Cell 157, 651-663 (2014)| Karaca, E. et al. Human CLP1 mutations alter tRNA biogenesis, affecting both peripheral and central nervous system function. Cell 157, 636-650 (2014) 